\title{
BMJ Open The relationship between time perspective and self-regulatory processes, abilities and outcomes: a protocol for a meta-analytical review
}

\author{
Harriet M Baird, Thomas L Webb, Jilly Martin, Fuschia M Sirois
}

To cite: Baird HM, Webb TL, Martin J, et al. The relationship between time perspective and self-regulatory processes, abilities and outcomes: a protocol for a metaanalytical review. BMJ Open 2017;7:e017000. doi:10.1136/ bmjopen-2017-017000

- Prepublication history and additional material are available. To view these files please visit the journal online (http://dx.doi. org/10.1136/bmjopen-2017017000).

Received 27 March 2017 Revised 30 May 2017 Accepted 31 May 2017

Department of Psychology, University of Sheffield, Sheffield, UK

Correspondence to Harriet M Baird; harriet.baird@ sheffield.ac.uk

\begin{abstract}
Introduction Both theoretical and empirical evidence suggests that time perspective is likely to influence self-regulatory processes and outcomes. Despite the theoretical and practical significance of such relations, the relationship between time perspective and self-regulatory processes and outcomes across different measures, samples and life domains, including health, has yet to be explored.
\end{abstract}

Methods and analysis The proposed review will develop a taxonomy for classifying measures according to the selfregulatory process, ability or outcome that they are likely to reflect. Electronic scientific databases will be searched, along with relevant conference abstract booklets and citation lists. Additionally, a call for unpublished data will be submitted to relevant bodies. To be eligible for inclusion, studies must include a measure of time perspective and a measure of at least one self-regulatory process, ability and/ or outcome. Eligibility will not be restricted by publication date, language, type of sample or setting. The bivariate correlations will be extracted (or calculated) and submitted to a random-effects meta-analysis. The sampleweighted average effect size, heterogeneity, risk of bias and publication bias will be calculated, and the effects of categorical and continuous moderator variables on the effect sizes will be determined.

Ethics and dissemination The proposed meta-analysis will synthesise previously conducted research; thus, ethical approval is not required. The findings will be submitted for publication in an international peer-reviewed journal and reported as part of the first authorâ€ $€^{\mathrm{TM}} \mathrm{S}$ $\mathrm{PhD}$ thesis. The findings will also be disseminated to the research community and, where appropriate, to other interested parties through presentations at relevant academic and non-academic conferences.

A number of health-related problems arise as a direct result of people's decisions to engage in particular behaviours. For example, statistics suggest that approximately two-thirds of premature deaths in the UK are linked to people's behavioural choices and are, therefore, at least in part, avoidable. ${ }^{1}$ Nonetheless, people often behave in ways that are detrimental to their health. For example, while smoking has been identified as the biggest

\section{Strengths and limitations of this study}

The proposed review will use existing theories to develop a taxonomy for classifying measures according to the self-regulatory process, ability or outcome that they are likely to reflect. This will allow us to investigate the influence of time perspective on specific self-regulatory processes, abilities and/ or outcomes.

- The proposed review will be inclusive and will not be restricted by the type of sample being studied, the study setting or the life domain being observed. Instead, these factors will be explored as potential moderators of the relationship between time perspective and self-regulatory processes, abilities and/or outcomes.

- Given the amount of primary evidence that is likely to be relevant for this review, it is possible that only a subsample of studies will be independently coded by a second reviewer. We will second code additional studies if agreement on this subsample is relatively low.

- Developing a taxonomy describing which measures reflect which self-regulatory processes and outcomes may enable future research to explore additional antecedents and consequences of selfregulatory processes and outcomes, and to conduct formal tests of self-regulation models.

behavioural risk factor for premature death, ${ }^{2}$ the prevalence rate of tobacco use in the UK is estimated to be as high as $20 \% .^{3}$ Similarly, although eating healthily and engaging in regular exercise is known to reduce the risk of cardiovascular disease and obesity, ${ }^{4}$ only $37 \%$ of adults in the UK achieve the recommended amount of physical activity ${ }^{5}$ and less than a third consume the recommended daily amount of fruit and vegetables. ${ }^{6}$ So, what determines whether people engage in behaviours that protect, rather than damage, their health?

Theoretical models of health behaviour (eg, the Theory of Planned Behaviour ${ }^{7}$; Social 
Cognitive Theory ${ }^{8}$; the Health Belief Model $^{9}$ ) recognise that the extent to which people consider the possible future outcomes of their actions is an important determinant of their subsequent behaviour and propose that behaviour is driven, in part, by weighing up the potential long-term benefits against the short-term rewards. For example, for a habitual smoker, having a cigarette is associated with a variety of immediate benefits, including feelings of pleasure, avoidance of withdrawal symptoms and improved concentration. However, in the long term, smoking is associated with a number of serious health-related problems, including lung cancer, cardiovascular disease and premature death. ${ }^{10}$ As such, the extent to which a person values future benefits over immediate pleasures is considered to be an important factor in determining health behaviour. ${ }^{11}$

\section{TIME PERSPECTIVE}

In light of the above considerations, a growing body of research has explored whether and how individual differences in people's time perspective influence motivational and behavioural outcomes. Time perspective is conceptualised as a relatively stable individual difference in the extent to which people express attitudinal, attentional and behavioural preferences for the past, present or future. ${ }^{12}$ Over time, the evidence suggests that individuals come to develop a habitual focus on, or orientation towards, one time frame over anotherbe it reminiscing over the past, living for the moment or looking towards the future. These preferences have been found to serve as a cognitive bias that can affect people's judgements, decision-making and, ultimately, their behaviour. ${ }^{1213}$

Individual differences in time perspective have been explored in relation to a number of psychological constructs and behaviours, across a range of life domains. ${ }^{14}$ For example, a future time perspective (ie, the tendency to consider the future implications of present decisions and actions) has been associated with a variety of health-protective behaviours (eg, physical activity and healthy eating ${ }^{15}$ ), greater academic achievement, ${ }^{16}$ more responsible financial behaviours (eg, regular saving ${ }^{17}$ ) and pro-environmental behaviours (eg, water conserva$\operatorname{tion}^{18}$ ). In contrast, a present time perspective (ie, the tendency to make decisions and engage in behaviours that satisfy immediate needs and desires) has been associated with health risk behaviours (eg, substance use ${ }^{15}$ ), lower academic achievement, ${ }^{19}$ pathological gambling ${ }^{20}$ and risky driving. ${ }^{21}$ Although relatively less research has explored the influence of a past time perspective on psychological constructs and behaviours, there is some evidence to suggest that having a positive view of the past is associated with greater health responsibility ${ }^{22}$ and higher levels of education, ${ }^{23}$ while having a negative view of the past is associated with binge eating and drinking, ${ }^{24}$ and problematic internet use. ${ }^{25}$

\section{THE PROPOSED REVIEW}

A number of independent, empirical studies have explored the associations between time perspective and various processes, behaviours and outcomes relevant to self-regulation. Given the theoretical and practical significance of understanding these associations, synthesising current evidence on the relationship between time perspective and key self-regulatory processes and outcomes, across different measures, samples and life domains, is important. Thus, the aim of the proposed review is to quantify the size and direction of the relationship(s) between time perspective and self-regulatory processes and outcomes using meta-analysis. Previous meta-analyses have explored the relationship between time perspective and either a specific behaviour, such as procrastination, ${ }^{26}$ or a specific group of behaviours, such as environmental behaviours, ${ }^{27}$ positive health behaviours ${ }^{28} 29$ and occupational well-being. ${ }^{30}$ The proposed review aims to extend existing work by exploring the relationship between time perspective and specific self-regulatory processes and outcomes within the framework of a theoretical model (namely, Control Theory ${ }^{31}{ }^{32}$ ). In contrast to previous meta-analyses, the proposed review will not be restricted to a specific behaviour or life domain and will, instead, deconstruct the processes involved during self-regulation and integrate this research into a comprehensive and theory-driven model. To achieve this aim, a taxonomy will be created in order to classify measures according to the self-regulatory process, ability and/or outcome that they are likely to reflect.

\section{A TAXONOMY FOR CLASSIFYING MEASURES OF SELF- REGULATORY PROCESSES, ABILITIES AND OUTCOMES}

Self-regulation is the study of how people direct their thoughts, feelings and behaviours in order to achieve their goals. ${ }^{33}{ }^{34}$ As such, the term self-regulation is often used more broadly to describe the processes that are involved during goal striving. ${ }^{35}$ These processes can include deciding which goals to pursue, engaging in actions to pursue these goals, monitoring goal progress and warding off temptations or challenges that may derail goal pursuit. ${ }^{36}$ Given that self-regulation likely encompasses a number of processes, in order to provide an accurate estimation of the association with time perspective it is important that the core processes involved in self-regulation are identified to explore the unique influence of time perspective on each of these processes independently. Furthermore, due to the multidisciplinary nature of research into self-regulation, there has been considerable variation in how processes and behaviours relevant to self-regulation have been operationalised, measured and defined. ${ }^{34}$ As a result, problems can arise when trying to integrate findings across different disciplinary perspectives and methodological approaches.

In an effort to address these challenges, the proposed review will develop a framework for classifying measures according to the self-regulatory process, ability and/or 
outcome that they are likely to reflect. By doing so we aim to create a comprehensive framework that can be used to organise and synthesise research on self-regulation, both in relation to time perspective (as in the proposed review), but also in subsequent research that explores other antecedents and consequences of self-regulatory processes and/or outcomes. Indeed, the development of this taxonomy may be particularly beneficial for researchers who are interested in health behaviours or the role of self-regulation in the self-management of certain medical conditions. For example, understanding the relative impact of time perspective on specific self-regulatory processes and abilities, and, in turn, their impact on health outcomes, may inform strategies and interventions designed to target certain self-regulatory processes (and the factors that influence them) in order to promote people's health and well-being.

\section{A FRAMEWORK FOR UNDERSTANDING SELF-REGULATION}

The proposed review will build on a framework for understanding self-regulation developed by Burnette and colleagues $^{38}$ and will distinguish between three self-regulatory processes as specified by Control Theory ${ }^{31}{ }^{32}$; namely, goal setting, goal monitoring and goal operating. In addition to investigating the impact of time perspective on specific self-regulatory processes, we will also examine the influence of time perspective on people's ability to regulate their behaviour (ie, their self-regulatory ability) and on the outcomes of their goal striving (ie, self-regulatory outcomes). Below, we outline the components of Control Theory identified above and suggest how individual differences in people's time perspective may influence their engagement in these self-regulatory processes and their ability to regulate their behaviour.

While a number of models of self-regulation have been proposed, Control Theory ${ }^{31} 32$ is considered one of the most influential models to date. ${ }^{39}$ Control Theory starts with the assumption that goal striving requires that people establish a specific reference value or a desired outcome; that is, the goal that they would like to achieve (this is the process of goal setting). According to Control Theory, once a goal has been formed, a process of monitoring is initiated in which people compare how things are (ie, their current rate of progress) with how they want or expect them to be (ie, the standard specified by the goal). If a discrepancy is detected, then people can respond by either taking goal-directed action or by revising their goal (this is the process of goal operating). As such, Control Theory suggests that goal striving involves three process: (i) setting goals, (ii) monitoring progress towards these goals and (iii) acting to reduce discrepancies.

\section{THE RELATIONSHIP BETWEEN TIME PERSPECTIVE AND SELF-REGULATION}

Both theoretical and empirical evidence suggests that time perspective is likely to influence the processes involved in self-regulation. Indeed, previous research has indicated that individuals with a future time perspective have stronger intentions to achieve their goals, ${ }^{40-42}$ are more likely to monitor their goal progress (Baird, Webb, Martin, et al., 2015, unpublished manuscript; Webb, 2014, unpublished data) and engage in goal-directed behaviours, such as physical activity and healthy eating ${ }^{43}$ studying, ${ }^{16}$ planning for retirement ${ }^{44}$ and managing finances. ${ }^{17}$ However, it is currently unclear which (if any) of the self-regulatory processes account for the likely effect of time perspective on self-regulatory outcomes. For example, do people with a future time perspective have better outcomes because they are (i) more motivated, (ii) more likely to keep track of their behaviour and/or (iii) more likely to take action when needed?

In addition to exploring the relationship between time perspective and specific self-regulatory processes, the proposed review will also explore the relationship between time perspective and people's ability to regulate their behaviour. Self-regulatory ability can be broadly defined as the resources and attributes that an individual has in order to help them with goal attainment. ${ }^{45}$ One self-regulatory ability that has gained a considerable amount of research attention is self-control, which is defined as the ability to inhibit behaviours that may derail goal pursuit, ${ }^{344-47}$ and enables an individual to prioritise their long-term over their short-term goals. ${ }^{35}$ Self-regulatory ability may also be reflected in measures of executive functioning, which reflect a number of goal-directed, cognitive processes (eg, working memory, behavioural inhibition and task switching) that enable conscious control over thoughts, feelings and behaviours. ${ }^{48}$ Thus, a further aim of the proposed review is to explore whether self-regulatory ability may account for the proposed relationship between time perspective and self-regulatory processes and/or outcomes.

\section{WHAT FACTORS MIGHT INFLUENCE THE RELATIONSHIP BETWEEN TIME PERSPECTIVE AND SELF-REGULATION?}

While greater consideration of future consequences is generally found to be associated with positive behavioural outcomes in a variety of life domains, the findings of some empirical studies are inconsistent with this conclusion. As such, it is important to try to identify factors that might influence the relationship between time perspective and self-regulation. One potential candidate is the nature of the measure of time perspective. For example, Adams and Nettle ${ }^{49}$ found that, while the future subscale of the Consideration for Future Consequences scale ${ }^{50}$ was negatively associated with smoking behaviour, the future subscales of other measures of time perspective (eg, the Zimbardo Time Perspective Inventory ${ }^{12}$ ) were not. Additionally, evidence suggests that the relationship between time perspective and some behaviours can vary according to the sample being studied. For example, while a future time perspective has been found to be associated with greater physical activity and a healthy diet among a sample of adolescents, ${ }^{43}$ a future time perspective was not 
associated with either physical activity or diet in a sample of patients undergoing cardiac rehabilitation. ${ }^{22}$ Thus, a final aim of the proposed review is to try to address empirical ambiguities by identifying and evaluating the impact of potential moderators on the relationship between time perspective and self-regulatory processes, abilities and outcomes.

\section{OBJECTIVES}

The proposed review will use meta-analysis to quantify the size of the relationships between time perspective and three key self-regulatory processes as specified by Control Theory: (i) goal setting, (ii) goal monitoring and (iii) goal operating. In addition to these processes, the proposed review will also include measures of self-regulatory ability and self-regulatory outcomes, and explore their associations with time perspective. These associations will be examined across a number of different domains (eg, health, academic, work, financial, environmental), samples (eg, community, students, adolescents) and countries.

To this end, the proposed review has four broad aims:

1. to use existing theories and frameworks to develop a taxonomy for classifying measures according to whether they reflect one or more of the self-regulatory processes described above, self-regulatory ability and/ or self-regulatory outcomes;

2. to use meta-analysis to quantify the direction and strength of the relationships between time perspective and self-regulatory processes, ability and outcomes;

3. to test whether the relationship between time perspective and self-regulatory outcomes is mediated (or explained) by the effect of time perspective on self-regulatory processes and/or ability;

4. to explore the theoretical and methodological moderators that influence the magnitude of these relationships.

\section{Method}

This protocol has been drafted using guidance from the Preferred Reporting Items for Systematic Reviews and Meta-Analyses Protocols (PRISMA-P ${ }^{51}$; see online supplementary material A), and with respect to recent recommendations for the meta-analysis of correlational data. ${ }^{52}$ The protocol has also been registered with the International Prospective Register of Systematic Reviews (PROSPERO, http://www.crd.york.ac.uk/PROSPERO; registration number: CRD42017058590).

\section{INCLUSION CRITERIA}

\section{Type of studies}

The proposed review will include published and unpublished empirical studies that examine the relationship between time perspective and (at least one) self-regulatory process, ability and/or outcome. Studies that adopt a correlational (eg, cross-sectional or longitudinal) or experimental design (ie, where time perspective is manipulated) will both be eligible for inclusion. In each case, the effect size $r$ will be used to represent the strength and direction of the relationship between time perspective and self-regulatory processes, abilities and/or outcomes. The design of the study (ie, correlational vs experimental) will be explored as a moderator in the analyses to examine whether the design of the studies influences the relationships observed.

\section{Sample}

No restriction will be placed on the type of sample being studied and, as such, studies of healthy adults, adolescents and children, university students and clinical populations will all be eligible for inclusion. The type of sample will be coded and included as a moderator in the analyses to explore whether the type of sample influences the relationship between time perspective and self-regulation.

\section{Measures of interest}

To be eligible for inclusion, studies must include a measure of time perspective and a measure of a self-regulatory process (ie, goal setting, goal monitoring or goal operating), ability or outcome. Below, we explain how we conceptualise each variable and plan to operationalise this inclusion criterion.

\section{Measures of time perspective}

The proposed review will conceptualise time perspective as a multidimensional construct that encompasses cognition, attention, attitude and behaviour. As such, our definition of time perspective will be broad and will include measures of time perspective such as the Zimbardo Time Perspective Inventory, ${ }^{12}$ as well as measures of future orientation (eg, the Consideration of Future Consequences Scale $\left.{ }^{50}\right)$, temporal depth (eg, the Temporal Depth Index ${ }^{53}$ ), temporal focus (eg, the Temporal Focus Scale ${ }^{54}$ ) and time attitudes (eg, the Time Attitude Scale ${ }^{55}$ ). We will also include measures of time perspective that have been used in specific samples (eg, the Hypertension Temporal Orientation Scale ${ }^{56}$ ) and scales in which time perspective is measured as a subscale (eg, the present-hedonistic subscale of the Barrett Impulsivity Scale ${ }^{57}$ ), assuming that it is possible to extract the bivariate correlation between the subscale reflecting time perspective and the measure of self-regulatory process, ability and/or outcome.

\section{Measures of self-regulation}

Studies must also include a measure of a self-regulatory process (ie, goal setting, goal monitoring or goal operating), ability or outcome. Given that there is considerable variation in how these processes and behaviours have been operationalised and defined, ${ }^{34}$ the proposed review will use existing theories (eg, Control Theory ${ }^{31}$ ) and frameworks ${ }^{385859}$ to develop a taxonomy for classifying measures according to the self-regulatory process that they are likely to reflect. To achieve this aim, a coding manual has been drafted by the review authors (see online supplementary 
material B). This manual includes a definition of each self-regulatory process, along with self-regulatory ability and outcomes, an example of how constructs relevant to each component are typically measured, and instructions for coders to classify measures. Two independent researchers working in the field of self-regulation will be provided with the coding manual. They will be asked to consider each measure of self-regulation extracted from eligible studies and indicate whether they think that the measure reflects a self-regulatory process (ie, setting, monitoring or operating), ability or outcome. Alternatively, they will be able to indicate that they do not think that the measure is relevant to self-regulation.

\section{Goal setting}

Goal setting typically reflects the amount of effort or motivation that a person will devote to achieving a goal. ${ }^{7}$ As such, measures of goal setting may include measures of intentions (eg, 'I intend to achieve $\mathrm{X}^{, 60}$ ), commitment or motivation (eg, 'I am strongly committed to achieving $\mathrm{X}^{, 61}$ ) or readiness to change (eg, precontemplation, contemplation, preparation, action and maintenance ${ }^{62}$ ).

\section{Goal monitoring}

Goal monitoring involves evaluating ongoing performance relative to the goal that has been set, or in comparison to others or past performance. ${ }^{32}{ }^{63}$ Example measures may include the frequency with which people check their personal finances, ${ }^{64}$ seek feedback on their performance at work (eg, from supervisors or colleagues ${ }^{65}$ ), record the number of calories consumed ${ }^{66}$ or use equipment that provides information relating to their goal progress (eg, smartphone apps, activity wristbands, weighing scales ${ }^{67}$ ).

\section{Goal operating}

Goal operating refers to activities and behaviours directed towards goal achievement. ${ }^{32}$ Measures of goal operating may include the number of hours spent preparing for an exam, ${ }^{16}$ planning for retirement (eg, enquiring about a saving scheme ${ }^{68}$ ), use of learning strategies (eg, reading the information several times or creating mnemonics ${ }^{69}$ ) or the amount of engagement or effort exerted towards a particular goal (eg, at work or while studying ${ }^{70}$ ).

\section{Self-regulatory ability}

Self-regulatory ability is conceptualised as the resources and attributes that an individual has in order to help them to achieve their goals. ${ }^{45}$ Therefore, measures of self-regulatory ability may include measures of self-control (eg, the Brief Self-Control Scale ${ }^{71}$ ), delay discounting ${ }^{72}$ impulsivity and sensation seeking (eg, the Barrett Impulsivity Scale ${ }^{57}$; the Sensation-Seeking Scale ${ }^{73}$ ), executive function (eg, response inhibition ${ }^{74}$ ) and problem-solving ability (eg, the Raven Advanced Progressive Matrices ${ }^{75}$ ). Measures of emotion regulation may also be relevant, including measures of competence at emotion regulation, ${ }^{76}$ proactive coping (eg, Proactive Coping Inventory ${ }^{77}$ ), ability to suppress aggression (eg, the suppression of aggression subscale of the Weinberger Adjustment Inventory ${ }^{78}$ ) and emotional stability (eg, the emotional stability subscale of the Ten Item Personality Inventory ${ }^{79}$ ).

\section{Self-regulatory outcomes}

Self-regulatory outcomes refer to the outcome(s) of goal pursuit (ie, what has been achieved). However, often researchers make inferences about the types of goals that people have. For example, it may be (wrongly) assumed that smokers have the goal to quit or reduce their smoking behaviour. In order to account for these assumptions, the proposed review will code measures of self-regulatory outcomes according to the level of inference that needs to be made about the degree to which the outcome reflects goal achievement (ie, how likely it is that people would want to achieve the goal). This could range from little or no inference; for example, when a study has specified a particular goal (eg, a study of smokers who want to stop smoking completely in 2 weeks $^{80}$ ) to high inference (eg, a study on alcohol consumption in a sample of adults who did not specify whether or not they wanted to reduce their alcohol consumption ${ }^{81}$ ). To ensure transparency and replicability, a coding framework will be developed listing the measures of self-regulatory outcomes extracted from eligible studies. Each measure will then be independently coded according to the level of inference that is being made (eg, participants' goal is clearly specified, low inference, medium inference or high inference). The level of inference will then be explored as a potential moderator of the relationship between time perspective and self-regulatory outcomes.

Possible measures of self-regulatory outcomes may include students' grade point averages, ${ }^{82}$ smokers' carbon monoxide levels, ${ }^{83}$ the amount of savings or debt that people have accumulated ${ }^{84}$ or a person's body mass index. ${ }^{67}$ Measures of self-regulatory outcomes may also include whether or not people engage in health-protective behaviours (eg, physical activity, health screenings, medication adherence ${ }^{15}{ }^{85}$ ) or health risk behaviours (eg, substance use, alcohol consumption, risky sexual behaviours, consuming fatty foods ${ }^{86}$ ), and the extent to which people engage in pro-environmental behaviours, risky driving or antisocial behaviours (eg, gambling, violence or expressions of aggression ${ }^{2087}$ ).

\section{Methodological design}

The proposed review will incorporate studies with both experimental and correlational designs. Correlational studies will be coded as 'cross-sectional' when the measure of time perspective and self-regulatory process, ability and/or outcome are taken at the same time. Correlational studies will be coded as 'longitudinal' when the measure of self-regulatory process, ability and/or outcome is taken after the measure of time perspective. The time interval between the measures of time perspective and self-regulatory process(es), ability or outcomes will also be coded according to the number of weeks between these measures. For example, the time interval in cross-sectional studies will be coded as ' 0 ', while longitudinal studies will be coded as 
' 1 ', '2' or ' 3 ' to represent a follow-up interval of 1, 2 or 3 weeks, respectively. For experimental studies, where time perspective is manipulated, the number of weeks between the manipulation and the measure(s) of self-regulatory processes, abilities and/or outcomes will be coded. If a measure of a self-regulatory process, ability or outcome is taken at multiple time points, then the data from the longest follow-up point will be extracted. The number of weeks between the measure of time perspective and the subsequent measure of self-regulation will be included as a moderator in the analyses in order to explore whether the length of follow-up influences the observed relations.

\section{Setting}

There will be no restrictions by the type of study setting, and we plan to include studies conducted in community, clinical and academic settings. The type of study setting will be coded and explored as a moderator in the analyses.

\section{Language}

No language restrictions will be imposed for this review; however, only studies that can be adequately translated, for example, through computer applications (eg, Google Translate) or by using transcription services available at the host institution, will be included. A record will be kept of potentially eligible studies that we were unable to translate.

\section{Information sources and search strategy}

Five strategies will be used to generate the sample of studies. First, a computerised search of two scientific electronic databases (Web of Knowledge and ProQuest Dissertation \& Theses) will be conducted for empirical articles published prior to the date of the search, using search terms relating to time perspective (ie, time perspective, time orientation, time attitude, temporal perspective, temporal orientation, temporal focus, temporal depth, future consequence, past orient ${ }^{*}$, present orient ${ }^{*}$, future orient ${ }^{*}$ ). Articles must include one or more of the search terms in the title, abstract or keywords.

Second, articles cited in the appendix of a recently published book, Time perspective theory; review, research and application, ${ }^{14}$ and articles listed on the reference page of the Time Perspective Network website (http://www.timeperspective.net/) will be reviewed for inclusion. Third, abstract booklets from the three International Conferences on Time Perspective that have been held to date will be screened for unpublished and published articles. Fourth, an email will be sent to the distribution lists of the European Association of Social Psychology (http:// www.easp.eu/) and members of the Time Perspective Network (http://www.timeperspective.net/) requesting relevant in-press or unpublished studies that might not otherwise have been identified by our searches. Finally, the reference lists of included articles will be checked for citations to other potentially relevant studies (ie, ancestry $\left.\operatorname{approach}^{88}\right)$.

\section{STUDY RECORDS}

\section{Data management}

Records identified via the electronic database searches will be exported into an EndNote Library (Endnote X7, Thomson Reuters, San Francisco, California, USA) for screening and the removal of duplicates. Studies retrieved via other search strategies will be entered into a Microsoft Excel spreadsheet. These studies will be manually crosschecked with the EndNote Library in order to identify any remaining duplicates. Once screening is complete, a data extraction form (see online supplementary material C for a draft) will be used to detail the relevant information from eligible studies. The data will then be exported into the statistics package $\mathbf{R}^{89}$ for analysis.

\section{Selection process}

The process of identifying relevant studies will be conducted in two stages. First, the first author (HMB) will screen the titles and abstracts of articles identified via the search strategy described above to identify potentially relevant studies. Second, the first author will review the full texts of articles describing potentially relevant studies against the inclusion and exclusion criteria. A random sample of these studies (approximately 20\%) will also be independently reviewed by the second author (TLW). Disagreements will be resolved through consultation with additional reviewers (JM and FMS), and if necessary, further information will be sought from the authors of the article in question in order to resolve any discrepancies. The reason(s) for excluding a study during the full-text review will be recorded and reported in a PRISMA diagram, ${ }^{90}$ showing the flow of studies through the review. It will not be feasible for reviewers to be blind to the study authors, journal or institutions.

\section{Data collection}

The data will be extracted using the data extraction form by the first author (HMB). A random sample of articles (approximately 20\%) will also be independently coded by the second author (TLW). Prior to data extraction, the form will be piloted using a sample of eligible studies to assess and refine the form as needed. Any disagreements that arise as a result of the data extraction will be resolved through discussion with additional reviewers (JM and FMS).

\section{DATA ITEMS}

The following information will be extracted from studies included in the review: (i) publication details (eg, authors, year of publication, publication status and language); (ii) sample characteristics (eg, mean age, gender composition and the type of sample being studied); (iii) methodological details, including the time interval (in weeks) between the measure of time perspective and the measure of self-regulatory process, ability or outcome, the country where the study was conducted, the measure(s) of time perspective and self-regulatory process(es), ability and/or outcome(s) 
used in the study, whether each measure of self-regulatory process, ability or outcome is measured objectively (eg, a smoker's carbon monoxide level) or via self-report (eg, a smoker's self-reported smoking frequency), reliability of the measures (test-retest, where reported; otherwise, measures of internal reliability, such as Cronbach's alpha) and the setting in which the study was conducted (eg, health, academic, financial, environmental); and (iv) statistical details, including the effect size (eg, Pearson's r statistic), how this effect size was calculated (see the section below for details of our approach to computing effect sizes) and the sample size associated with the effect size extracted.

\section{ASSESSMENT OF METHODOLOGICAL QUALITY AND RISK OF BIAS FROM INDIVIDUAL STUDIES}

A tailored quality assessment tool will be used in order to assess the quality of individual studies as recommended by Quintana. ${ }^{52}$ Specifically, a four-point assessment tool will be applied in which a point will be given for each of the following methodological properties. First, if the study uses a prospective design. Cross-sectional designs can inflate estimated effect sizes due to the simultaneous measurement of study variables, ${ }^{91}$ which can lead participants to modify their responses in order to appear consistent. ${ }^{92}{ }^{93}$ Second, an objective measure of self-regulatory process, ability and/or outcome is used. Objective measures reduce the influence of social desirability and recall biases. ${ }^{94} 95$ Third, a sample size $>85$ was recruited. A sample of 85 participants would have sufficient power (ie, $80 \%$ ) detect a medium effect size with an alpha of $0.05 .{ }^{96}$ Fourth, the measure of time perspective is internally reliable (ie, has a Cronbach's alpha of $\geq 0.70$ ). The risk of bias score will be included as a moderator in the analyses.

\section{DATA SYNTHESIS}

\section{Extracting effect sizes}

Studies will need to report, or provide sufficient information in order for us to be able to calculate, the bivariate Pearson's correlation ( $r$ ) representing the strength and direction of the relationship between the measure of time perspective and the measure of a self-regulatory process, ability or outcome. If required, an online converter (eg, Psychometrica ${ }^{97}$ ) will be used to convert other effect sizes (eg, d, f, eta-squared, OR) into $r$. If this information is not available from the original report, then the authors will be contacted and asked to provide this information. In cases where multiple measures of time perspective or self-regulatory process, ability or outcome are completed by the same sample at the same point in time, then a single composite effect size will be created by meta-analysing these effect sizes prior to inclusion in the main data set. If multiple samples are reported in the same study, then the effect sizes for each sample will be included as a separate data point, assuming that the procedures for each sample meet the inclusion criteria.

\section{Meta-analytic strategy}

Pearson's $r$ statistic will be used to represent the direction and strength of the relationship between the measures of time perspective and measures of self-regulation. However, as Pearson's $r$ is not normally distributed, all of the effect sizes will be converted to Fisher's z for analysis. These will then be converted back to Pearson's $r$ after analysis in order to report the (sample-weighted) average correlations and the associated 95\% CIs. The statistical software $R^{89}$ will be used to analyse the data, using the "metaphor' ${ }^{98}$ and 'robumeta' 99 packages. It is expected that the studies included in the proposed review will be drawn from different populations and, as such, a random-effects model will be applied in all analyses. This conservative approach assumes that the effect sizes may vary between studies and will allow the findings to be generalised to populations beyond the samples studied. ${ }^{100}$

\section{Sample heterogeneity}

The $\mathrm{I}^{2}$ and Q-statistic will be used to assess the heterogeneity of the effect sizes across studies. A statistically significant Q-statistic indicates that the effect sizes from the primary studies differ more than would be expected by chance alone and, therefore, that differences in the conceptual approach, methodology and/or sample may explain the heterogeneity. ${ }^{101}$ The $\mathrm{I}^{2}$ statistic reflects the proportion of the total variance that is attributable to between-study variability, and not due to sampling error within individual studies. The proposed meta-analysis will follow the convention proposed by Higgins and colleagues, with $25 \%, 50 \%$ and $75 \%$ representing low, medium and high variance, respectively. ${ }^{102}$

\section{Moderator analyses}

In addition to exploring the strength and direction of the relationships between measures of time perspective and measures of self-regulatory processes, abilities and outcomes, factors that might moderate these relationships will also be explored. Where the potential moderator is a continuous variable (eg, the age of the sample), a random-effects meta-regression model will be used to estimate the unstandardised regression coefficient and the significance of the coefficient. Where the potential moderator is categorical (eg, whether the measures are objective or self-reported), separate random-effects models will be conducted for each level of the moderator. The sample-weighted average effect sizes will then be compared using a two-tailed $\mathrm{z}$ test in order to determine whether they significantly differ, and thus, provide evidence of a moderation effect.

\section{META-BIASES}

\section{Assessment of publication bias}

A multimethod approach will be taken in order to assess publication bias. First, an initial inspection of the funnel plots will be conducted. Publication bias will then be formally assessed using Egger and colleagues' regression test ${ }^{103}$ and by calculating the fail-safe N. ${ }^{104}$ If there 
is evidence of publication bias, then Duval and Tweedie's trim and fill technique will be applied. ${ }^{105}$

\section{Amendments to protocol}

Any substantive amendment to this protocol will be registered with PROSPERO. If an amendment is made, then a description and rationale for the amendment will be provided in the final publication.

\section{Ethics and dissemination}

The proposed meta-analysis will synthesise the findings of previously conducted research and, therefore, ethical approval is not required. The findings will be submitted for publication in an international peer-reviewed journal and reported as part of a PhD thesis currently being conducted by the first author at the University of Sheffield in the UK. This thesis will also be uploaded to the White Rose Repository, although with an embargo pending publication of the findings in a peer-reviewed journal. The findings of the proposed review will also be disseminated to the research community and, where appropriate, to other interested parties (eg, policymakers) through presentations at relevant academic and non-academic conferences and via social media (eg, Twitter). If we believe that the findings are likely to be of interest to the wider public, then efforts will be made to disseminate the findings to the media via the press office at the University of Sheffield.

Contributors All authors contributed to the development of the search strategies, selection criteria, and data extraction procedures. HMB will conduct the searches and extract

Competing interests None declared.

Provenance and peer review Not commissioned; externally peer reviewed.

Open Access This is an Open Access article distributed in accordance with the Creative Commons Attribution Non Commercial (CC BY-NC 4.0) license, which permits others to distribute, remix, adapt, build upon this work non-commercially, and license their derivative works on different terms, provided the original work is properly cited and the use is non-commercial. See: http://creativecommons.org/ licenses/by-nc/4.0/

(c) Article author(s) (or their employer(s) unless otherwise stated in the text of the article) 2017. All rights reserved. No commercial use is permitted unless otherwise expressly granted.

\section{REFERENCES}

1. Department of Health. Living well for longer: a call to action to reduce avoidable premature mortality, 2013. Retrieved from. https:// www.gov.uk/government/uploads/system/uploads/attachment data/file/181103/Living_well_for_longer.pdf. ((accessed May 2017).).

2. Department of Health. Chief Medical Officer's Report. 2012. Retrieved From http://www.dh.gov.uk/health/2012/11/cmo/ ((accessed May 2017)).

3. Office for National Statistics. Adults smoking habits in Great Britain. 2014. Retrieved from http:// www.ons.gov.uk/peoplepopulationandcommunity/ healthandsocialcare/healthandlifeexpectancies/bulletins/ adultsmokinghabitsingreatbritain/2014 (accessed May 2017).

4. Buttar HS, Li T, Ravi N. Prevention of cardiovascular diseases: role of exercise, dietary interventions, obesity and smoking cessation. Exp Clin Cardiol 2005;10:229-49.

5. World Health Organisation. Global Health Observatory data repository. prevalence of insufficient physical activity among adults. 2010. Data by country. Retrieved from http://apps.who.int/gho/data/ node.main.A893?lang=en ((accessed May 2017).).
6. Health Survey for England. Trend tables. 2011. Retrieved from http://content.digital.nhs.uk/catalogue/PUB09302/HSE2011-Trendcommentary.pdf (accessed May 2017).

7. Ajzen I. The theory of planned behavior. Organ Behav Hum Decis Process 1991;50:179-211.

8. Bandura A. Social foundations of thought and action: a social cognitive theory. Englewood Cliffs, NJ: Prentice Hall, 1986.

9. Rosenstock IM. Historical origins of the Health Belief Model. Health Education \& Behavior 1974;2:328-35.

10. Centers for Disease Control and Prevention (2016). Tobacco-related mortality. Retrieved from. https://www.cdc.gov/tobacco/data_ statistics/fact_sheets/health_effects/tobacco_related_mortality/ index.htm. ((accessed May 2017)).

11. Conner M, Norman P. Predicting health behaviour. UK: McGraw-Hill Education, 2005.

12. Zimbardo PG, Boyd JN. Putting time in perspective: a valid, reliable individual-differences metric. J Pers Soc Psychol 1999;77:1271-88.

13. Boyd JN, Zimbardo PG. Time perspective health and risk taking. In: Strahman A, Joireman J, eds. Understanding behavior in the context of Time: theory, Research and applications. Mahwah, NJ: erlbaum, 2005:85-107.

14. Stolarski M, Fieulaine N, van Beek W. Time perspective theory; Review, research, and application. Cham, Switzerland: Springer International, 2015.

15. Daugherty JR, Brase GL. Taking time to be healthy: predicting health behaviors with delay discounting and time perspective. Pers Individ Dif 2010;48:202-7.

16. Horstmanshof L, Zimitat C. Future time orientation predicts academic engagement among first-year university students. $\mathrm{Br} J$ Educ Psychol 2007;77:703-18.

17. Donnelly G, lyer R, Howell RT. The big five personality traits, material values, and financial well-being of self-described money managers. J Econ Psychol 2012;33:1129-42.

18. Corral-Verdugo V, Fraijo-Sing B, Pinheiro JQ. Sustainable behavior and time perspective: present, past, and future orientations and their relationship with water conservation behavior. Interam J Psychol 2006;40:139-47.

19. Guthrie LC, Butler SC, Ward MM. Time perspective and socioeconomic status: a link to socioeconomic disparities in health? Soc Sci Med 2009;68:2145-51.

20. Hodgins DC, Engel A. Future time perspective in pathological gamblers. J Nerv Ment Dis 2002;190:775-80.

21. Zimbardo PG, Keough KA, Boyd JN. Present time perspective as a predictor of risky driving. Pers Individ Dif 1997;23:1007-23.

22. Hamilton JM, Kives KD, Micevski V, et al. Time perspective and health-promoting behavior in a cardiac rehabilitation population. Behav Med 2003;28:132-9.

23. Shores K, Scott D. The relationship of individual time perspective and recreation experience preferences. J Leis Res 2007;39:28-59.

24. Laghi F, Liga F, Baumgartner E, et al. Time perspective and psychosocial positive functioning among italian adolescents who binge eat and drink. J Adolesc 2012;35:1277-84.

25. Chittaro L, Vianello A. Time perspective as a predictor of problematic internet use: a study of Facebook users. Pers Individ Dif 2013;55:989-93.

26. Sirois FM. Out of sight, out of Time? A Meta-analytic investigation of Procrastination and Time Perspective. Eur J Pers 2014;28:511-20.

27. Milfont TL, Wilson J, Diniz P. Time perspective and environmental engagement: a meta-analysis. Int J Psychol 2012;47:325-34.

28. Sweeney AM, Culcea I. Does a future-oriented temporal perspective relate to body mass index, eating, and exercise? A meta-analysis. Appetite 2017;112:272-85.

29. Yarcheski A, Mahon NE, Yarcheski TJ, et al. A meta-analysis of predictors of positive health practices. J Nurs Scholarsh 2004;36:102-8.

30. Henry H, Zacher H, Desmette D. Future Time Perspective in the work context: a systematic review of quantitative studies. Front Psychol 2017;8.

31. Carver CS, Scheier MF. The self-attention-induced feedback loop and social facilitation. J Exp Soc Psychol 1981;17:545-68.

32. Carver CS, Scheier MF. Control theory: a useful conceptual framework for personality-social, clinical, and health psychology. Psychol Bull 1982;92:111-35.

33. de Ridder D, De Wit J. Self-regulation in health behavior. London, England: Wiley, 2006.

34. Vohs KD, Baumeister RF. Understanding self-regulation: An introduction. In: Baumeister RF, Vohs KD, eds. Handbook of selfregulation: research, Theory, and applications. New York: guilford, 2004:1-9. 
35. Fujita K. On conceptualizing self-control as more than the effortful inhibition of impulses. Pers Soc Psychol Rev 2011;15:352-66.

36. Fujita K, Carnevale JJ, Trope Y. Understanding Self-Control as a Whole vs. Part Dynamic. Neuroethics 2016;92:1.

37. Mann T, de Ridder D, Fujita K. Self-regulation of health behavior: social psychological approaches to goal setting and goal striving Health Psychol 2013;32:487-98.

38. Burnette JL, O'Boyle EH, VanEpps EM, et al. Mind-sets matter: a meta-analytic review of implicit theories and self-regulation. Psychol Bull 2013;139:655-701.

39. Johnson RE, Chang CH, Lord RG. Moving from cognition to behavior: what the research says. Psychol Bull 2006;132:381-415.

40. Crockett RA, Weinman J, Hankins M, et al. Time orientation and health-related behaviour: measurement in general population samples. Psychol Health 2009;24:333-50.

41. Fieulaine N, Martinez F. About the fuels of self-regulation: time perspective and desire for control in adolescents substance use. The Psychology of Self-Regulation 2011:102-21.

42. Griva F, Anagnostopoulos F, Potamianos G. Time perspective and perceived risk as related to mammography screening. Women Health 2013;53:761-76.

43. Luszczynska A, Gibbons FX, Piko BF, et al. Self-regulatory cognitions, social comparison, and perceived peers' behaviors as predictors of nutrition and physical activity: a comparison among adolescents in Hungary, Poland, Turkey, and USA. Psychol Health 2004:19:577-93.

44. Petkoska J, Earl JK. Understanding the influence of demographic and psychological variables on retirement planning. Psychol Aging 2009:24:245-51.

45. de Ridder DT, Lensvelt-Mulders G, Finkenauer C, et al. Taking stock of self-control: a meta-analysis of how trait self-control relates to a wide range of behaviors. Pers Soc Psychol Rev 2012;16:76-99.

46. Baumeister RF, Bratslavsky E, Muraven M, et al. Ego depletion: is the active self a limited resource? J Pers Soc Psychol 1998; $74: 1252-65$

47. Gillebaart M, de Ridder DTD. Effortless Self-Control: a Novel Perspective on Response Conflict strategies in Trait Self-Control. Soc Personal Psychol Compass 2015;9:88-99.

48. Hofmann W, Schmeichel BJ, Baddeley AD. Executive functions and self-regulation. Trends Cogn Sci 2012;16:174-80.

49. Adams J, Nettle D, perspective T. Time perspective, personality and smoking, body mass, and physical activity: an empirical study. $\mathrm{Br} J$ Health Psychol 2009;14:83-105.

50. Strathman A, Gleicher F, Boninger DS, et al. The consideration of future consequences: weighing immediate and distant outcomes of behavior. J Pers Soc Psychol 1994:66:742-52.

51. Moher D, Shamseer L, Clarke M, et al. Preferred reporting items for systematic review and meta-analysis protocols (PRISMA-P) 2015 statement. Syst Rev 2015:4:1-9.

52. Quintana DS. From pre-registration to publication: a non-technical primer for conducting a meta-analysis to synthesize correlational data. Front Psychol 2015;6:1-9.

53. Bluedorn AC. The human organization of time: temporal realities and experience. Stanford, CA: Stanford University Press, 2002.

54. Shipp AJ, Edwards JR, Lambert LS. Conceptualization and measurement of temporal focus: the subjective experience of the past, present, and future. Organ Behav Hum Decis Process 2009;110:1-22.

55. Nuttin J. Future Time Perspective and motivation. Hillsdale, NJ: Lawrence Erlbaum, 1985

56. Brown CM, Segal R. The development and evaluation of the hypertension temporal orientation (HTO) scale. Ethn Dis 1997;7:41-54.

57. Patton JH, Stanford MS. Factor structure of the Barratt impulsiveness scale. J Clin Psychol 1995:51768-74.

58. Harkin B, Webb TL, Chang BP, et al. Does monitoring goal progress promote goal attainment? A meta-analysis of the experimental evidence. Psychol Bull 2016;142:198-229.

59. Sirois FM, Molnar DS, Hirsch JK et al. A Meta-analytic and Conceptual Update on the associations between Procrastination and Multidimensional perfectionism. Eur J Pers 2017:31:137-59.

60. Triandis HC. Values, attitudes, and interpersonal behavior. In: Howe HE, Page M, eds. Nebraska Symposium of motivation. Lincoln: university of Nebraska Press, 1980:195-259.

61. Locke EA, Latham GP, Erez M. The determinants of goa commitment. Acad Manage Rev 1988;13:23-39.

62. Prochaska JO, DiClemente CC. Self change processes, self efficacy and decisional balance across five stages of smoking cessation. Prog Clin Biol Res 1984;156:131-40.
63. Webb TL, Chang BPI, Benn Y. 'The Ostrich Problem': Motivated Avoidance or Rejection of Information About Goal Progress. Soc Personal Psychol Compass 2013;7:794-807.

64. Chang BPI, Webb TL, Benn Y, et al. Monitoring personal finances: evidence that goal progress and regulatory focus influence when people check their balance. J Econ Psychol. In Press. 2017.

65. Anseel F, Lievens F, Levy PE. A self-motives perspective on feedback-seeking behavior: linking organizational behavior and social psychology research. International Journal of Management Reviews 2007:9:211-36.

66. Boutelle KN, Kirschenbaum DS, Baker RC, et al. How can obese weight controllers minimize weight gain during the high risk holiday season? by self-monitoring very consistently. Health Psychol 1999;18:364-8

67. Hall PA, Epp L. Does domain-specific time perspective predict accelerometer assessed physical activity? an examination of ecological moderators. Psychol Sport Exerc 2013;14:50-6.

68. Hershey DA, Jacobs-Lawson JM, McArdle JJ, et al. Psychological foundations of Financial Planning for Retirement. J Adult Dev 2007:14(1-2):26-36.

69. Bowles T. The relationship of time orientation with perceived academic performance and preparation for assessment in adolescents. Educ Psychol 2008;28:551-65.

70. Gutiérrez-Braojos C. Future time orientation and learning conceptions: effects on metacognitive strategies, self-efficacy beliefs, study effort and academic achievement. Educ Psychol 2015;35:192-212.

71. Tangney JP, Baumeister RF, Boone AL. High self-control predicts good adjustment, less pathology, better grades, and interpersonal success. J Pers 2004;72:271-324.

72. Mischel W, Shoda Y, Rodriguez MI. Delay of gratification in children Science 1989;244:933-8

73. Kolin EA, Price L, ZOOB I, et al. Development of a sensationseeking scale. J Consult Psychol 1964;28:477-82.

74. Logan GD, Cowan WB, Davis KA. On the ability to inhibit simple and choice reaction time responses: a model and a method. J Exp Psychol Hum Percept Perform 1984;10:276-91.

75. Raven J, Raven JC, Court JH. Manual for Raven's Advanced Progressive Matrices. Oxford, England: Oxford Psychologists Press, 1998.

76. Niven K, Totterdell P, Miles E, et al. Achieving the same for less: improving mood depletes blood glucose for people with poor (but not good) emotion control. Cogn Emot 2013;27:133-40.

77. Greenglass E, Schwarzer R, Jakubiec D, et al. The proactive coping inventory (PCI): A multidimensional research instrument.1999. Jan 23, 2017. Retrieved from http://estherg.info.yorku.ca/files/2014/09/ pci.pdf ((accessed May 2017))

78. Weinberger DA. Distress and self-restraint as measures of adjustment across the life span: confirmatory factor analyses in clinical and nonclinical samples. Psychol Assess 1997;9:132-5.

79. Gosling SD, Rentfrow PJ, Swann WB. A very brief measure of the Big-Five personality domains. J Res Pers 2003;37:504-28.

80. Beenstock J, Lindson-Hawley N, Aveyard P, et al. Future orientation and smoking cessation: secondary analysis of data from a smoking cessation trial. Addiction 2014;109:1732-40.

81. Carey KB, Henson JM, Carey MP, et al. Which heavy drinking college students benefit from a brief motivational intervention? $J$ Consult Clin Psychol 2007;75:663-9.

82. Shell DF, Husman J. The multivariate dimensionality of Personal Control and Future Time Perspective Beliefs in Achievement and Self-Regulation. Contemp Educ Psychol 2001;26:481-506.

83. Jones BA, Landes RD, Yi R, et al. Temporal horizon: modulation by smoking status and gender. Drug Alcohol Depend 2009;104 Suppl 1:S87-S93.

84. Antonides G, Manon de Groot I, Fred van Raaij W, De Groot IM, Van Raaij WF. Mental budgeting and the management of household finance. J Econ Psychol 2011;32:546-55.

85. Sansbury B, Dasgupta A, Guthrie L, et al. Time perspective and medication adherence among individuals with hypertension or diabetes mellitus. Patient Educ Couns 2014:95:104-10.

86. Henson JM, Carey MP, Carey KB, et al. Associations among health behaviors and time perspective in young adults: model testing with boot-strapping replication. J Behav Med 2006;29:127-37.

87. McKay MT, Dempster M, Mello Z. Does consideration of future consequences moderate the relationship between aggression and alcohol use in adolescents? results from the United Kingdom Addict Res Theory 2015;23:372-9.

88. Johnson BT. D. Software for the meta-analytic review of research literatures. Hillsdale, NJ: Erlbaum, 1993.

89. Core Team R. R: a language and environment for statistical computing. R Foundation for Statistical Computing, Vienna, Austria 
2013. Retrieved from http://www.R-project.org/ (accessed May 2017).

90. Moher D, Liberati A, Tetzlaff J, et al. Preferred reporting items for systematic reviews and meta-analyses: the PRISMA statement. $J$ Clin Epidemiol 2009;62:1006-12.

91. Lindell MK, Whitney DJ. Accounting for common method variance in cross-sectional research designs. J Appl Psychol 2001;86:114-21.

92. Budd RJ. Response Bias and the Theory of Reasoned Action. Soc Cogn 1987;5:95-107.

93. Fishbein M, Ajzen I. Predicting and changing behavior: the reasoned action approach. New York, NY: Psychology Press, 2010.

94. Hassan E. Recall Bias can be a threat to retrospective and prospective research designs. The Internet Journal of Epidemiology 2006;3:339-412.

95. Nederhof AJ. Methods of coping with social desirability Bias: a review. Eur J Soc Psychol 1985;15:263-80.

96. Cohen J. A power primer. Psychol Bull 1992;112:155-9.

97. Lenhard W, Lenhard A. Calculation of effect sizes. Retrieved from https://www.psychometrica.de/effect_size.html (accessed May 2017).
98. Viechtbauer W. Conducting Meta-Analyses in $R$ with the metafor Package. J Stat Softw 2010;36:1-48.

99. Fisher Z, Tipton E. Robumeta: An R-package for robust variance estimation in meta-analysis. Retrieved from. http://blogs.cuit. columbia.edu/let2119/files/2013/03/ Fisher-and-Tipton-.pdf. (accessed May 2017).

100. Hedges LV, Olkin I. Statistical methods for meta-analysis. Orlando, FL: Academic press, 1985.

101. Shadish WR, Haddock CK. Combining estimates of effect size. In: Cooper H, Hedges LV, Valentine JC, eds. The handbook of Research synthesis and Meta-analysis. New York, NY: Russell Sage Foundation, 2009:257-77.

102. Higgins JP, Thompson SG, Deeks JJ, et al. Measuring inconsistency in meta-analyses. BMJ 2003;327:557-60.

103. Egger M, Davey Smith G, Schneider M, et al. Bias in meta-analysis detected by a simple, graphical test. BMJ 1997;315:629-34.

104. Rosenthal R. The file drawer problem and tolerance for null results. Psychol Bull 1979;86:638-41.

105. Duval S, Tweedie R. Trim and fill: a simple funnel-plot-based method of testing and adjusting for publication Bias in metaanalysis. Biometrics 2000;56:455-63. 